\title{
HER2-positive double primary tumor of gastric and breast cancer occur synchronously in a patient: A case report
}

\author{
QUCHANG OUYANG $^{1 *}$, CAN TIAN ${ }^{1}$, JIANXIANG GAO $^{1}$, JIN HUANG $^{2}$, \\ $\mathrm{HUA} \mathrm{FU}^{3}$, JINSONG $\mathrm{HE}^{4 *}$ and JIANBO YANG ${ }^{1,5}$ \\ ${ }^{1}$ Oncology Division of Breast Cancer, Hunan Cancer Hospital, The Affiliated Cancer Hospital of Xiangya School of Medicine, \\ Central South University, Changsha, Hunan 410013; ${ }^{2}$ Shanghai Roche Pharmaceuticals, Pudong, Shanghai 201203; \\ ${ }^{3}$ The Third Affiliated Hospital of Xiangya School of Medicine, Central South University, Changsha, Hunan 410013; \\ ${ }^{4}$ Department of Breast Surgery, The First Affiliated Hospital of Shenzhen University, The Second People's Hospital \\ of Shenzhen, Shenzhen, Guangdong 518029, P.R. China; ${ }^{5}$ Department of Laboratory Medicine and Pathology, \\ Masonic Cancer Center, University of Minnesota, Minneapolis, MN 55455, USA
}

Received September 8, 2015; Accepted February 22, 2016

DOI: $10.3892 / \mathrm{mco} .2016 .812$

\begin{abstract}
The simultaneous occurrence of primary gastric cancer and breast cancer is rare, and the positive expression of human epidermal growth factor receptor (HER)2 in double primary carcinoma of gastric and breast cancer remains to be reported. The present study presented a 46-year-old woman complaining of irregular acid reflux and stomach discomfort. The stomach cancer was diagnosed by esophagogastroduodenoscopy examination of the pathological biopsies in 2010 . The patient underwent a radical gastrectomy for gastric cancer, and postoperative pathological examination revealed moderately-poorly differentiated adenocarcinoma with HER2 positive expression. The tumor invaded into the entire thickness of the gastric wall and lymph nodes. The patient received five treatments of postoperative chemotherapy. In August 2011, the patient felt a lump in the right breast. Simple excision of the right breast mass was performed on September 2011, and postoperative pathological examination revealed the invasive ductal carcinoma of the right breast with HER2 amplification by fluorescent in situ hybridization assay. The patient was treated with postoperative chemotherapy and radiotherapy, and also Trastuzumab target therapy. The patient succumbed to aggressive disease progression in March 2012.
\end{abstract}

Correspondence to: Dr Jianbo Yang or Dr Quchang Ouyang, Oncology Division of Breast Cancer, Hunan Cancer Hospital, The Affiliated Cancer Hospital of Xiangya School of Medicine, Central South University, 283 Tong Zi Po Road, Yuelu, Changsha, Hunan 410013, P.R. China

E-mail: Jianbo_yang@hotmail.com

E-mail: oyqc1969@126.com

${ }^{*}$ Contributed equally

Key words: double primary carcinoma, breast cancer, gastric cancer, HER2 positive

\section{Introduction}

Double primary carcinoma refers to the simultaneous occurrence of different primary histological malignancies in different parts of the body and belongs to the category of multiple primary cancers $(1,2)$. The survival of patients with breast cancer has greatly improved with earlier screening and improvement of treatment. Accordingly, the incidence of the second or multiple primary malignancies among breast cancer patients has been found to increase. In clinical practice, the second or multiple primary malignancies are very frequently indistinct from the metastases of the first primary malignancy, leading to the misdiagnosis and improper treatment for patients with double primary carcinoma. The simultaneous occurrence of primary gastric cancer and breast cancer is extremely rare.

Overexpression of HER2 is a frequent molecular abnormality in primary breast cancer and primary gastric cancer. To the best of our knowledge, no patient with HER2 positivedouble primary carcinoma in the gastrointestinal system and the breast has been reported previously. The present study reported one patient with double primary carcinoma of gastric and breast cancer.

\section{Case report}

A 46-year-old woman had complained of irregular acid reflux without obvious causes since August 2010. This patient received no treatment. On November $25^{\text {th }} 2010$, a Helicobacter pylori test was performed and the result was positive. The patient was then treated with metronidazole + clarithromycin + omeprazole, however showed no significant improvement. The patient felt satiety after meals and occasionally exhibited symptoms of vague epigastric pain and hematemesis. On December $28^{\text {th }} 2010$, the patient underwent esophagogastroduodenoscopy (EGD) examination in The Third Affiliated Hospital of Xiangya Medical School (Guangdong, China). The results indicated abnormal changes of the gastric antrum, angular and mucous membranes, and stomach cancer was diagnosed following pathological 
biopsies. Since January 2011, the patient was treated with two courses of chemotherapy (regimens unknown) and referred to Hunan Cancer Hospital (Hunan, China) for further treatment. On February $18^{\text {th }} 2011$, the patient underwent a radical gastrectomy for gastric cancer. Postoperative pathological examination revealed moderately-poorly differentiated adenocarcinoma of the stomach which had invaded into the entire thickness of the gastric wall where vascular tumor thrombi were identified (Fig. 1). The involvement of the lymph nodes for $2 / 2$ in group $8 \mathrm{a} ; 1 / 1$ in group $9 ; 3 / 3$ in group $11 \mathrm{p} ; 1 / 1$ in group $7 ; 1 / 1$ in group 11v; $1 / 1$ in group $6 ; 4 / 4$ in lesser curvature group; $3 / 4$ in greater curvature group, and fiber fat tissues were identified in $12 \mathrm{a}, 12 \mathrm{~b}, 13$ and 15 group lymph nodes. The carcinoma invaded into the capsule of the pancreas and the carcinoma was not identified in duodenal stump, surgery margin and greater omentum.

The fluorescent in situ hybridization (FISH) assay of the HER2 gene in gastric cancer tissues is shown in Fig. 2. HER2 fluorescent signals were visualized as discrete punctuate staining dots. A total of 94 HER 2 signals and 46 chemosensory protein (CSP) 17 signals were detected in a total of 30 cells. The ratio of HER2/CSP17 was 2.043, and the final diagnosis was HER2 amplification and HER2 FISH-positive gastric cancer. The patient received five treatments of the postoperative chemotherapy regimen, docetaxel + Capecitabine + cisplatin, and the last treatment was Oteracil + Oxaliplatin.

In August 2011, a lump was felt in the right breast and the sonography revealed a hypoechoic mass $(18 \times 12 \mathrm{~mm})$ at the 10 o'clock position and $77 \mathrm{~mm}$ from the right breast nipple, $5 \mathrm{~mm}$ deep from the skin surface. The surrounding boundary was poorly defined with uneven internal echo, Ultrasound BI-RADS grade was 4A. A simple excision of the right breast mass was performed on September $1^{\text {st }} 2011$. Postoperative pathological examination (5 and 11 o'clock positions of the right breast) revealed an invasive ductal carcinoma of the right breast (Grade II-III), tumor thrombi were identified in the stromal vascular (5 o'clock position) and the local skin was invaded (Fig. 3). The tumor was estrogen receptor (-), progesterone receptor (-), CerbB-2 (++), Ki-67 labeling index rate of $70 \%$. A HER2 FISH assay revealed that fluorescent signals of the HER2 gene were visualized as punctate staining (Fig. 4). The total number of HER2 gene copies and CSP17 chromosome signals in the 30 counted cells was 163 and 72, respectively. The ratio of HER2/CSP17 was 2.28, and was indicated as HER2 amplification and FISH-positive for the HER2 gene. Postoperative chemotherapy was administered for one course (specific regimen unknown), followed by right chest wall + right axillary + right supraclavicular radiotherapy. In October 2011, the patient was admitted to Hunan Cancer Hospital (Hunan, China) for the first dose of Trastuzumab ( $8 \mathrm{mg} / \mathrm{kg}$ IV) target therapy, followed by two doses of Trastuzumab (6 mg/kg IV) cycled every 21 days at the outpatient clinic. The patient failed to adhere to the systemic treatment and did not respond to multiple of telephone follow-ups. The patient succumbed to aggressive disease progression in March 2012.

\section{Discussion}

Diagnostic criteria. Double primary cancers belong to the category of multiple primary cancers, referring to tumors

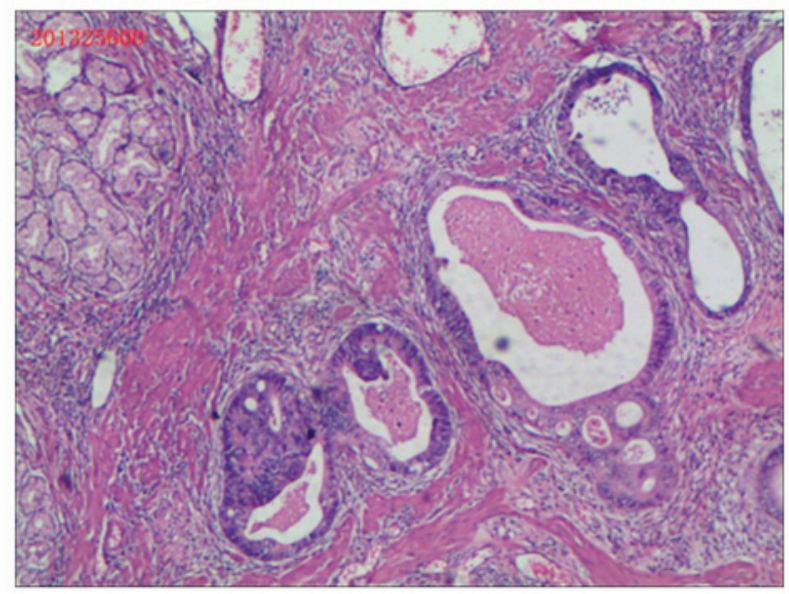

Figure 1. Photomicrographs of the stomach. Hematoxylin and eosin staining of the stomach revealed gastric cancer (magnification x100).

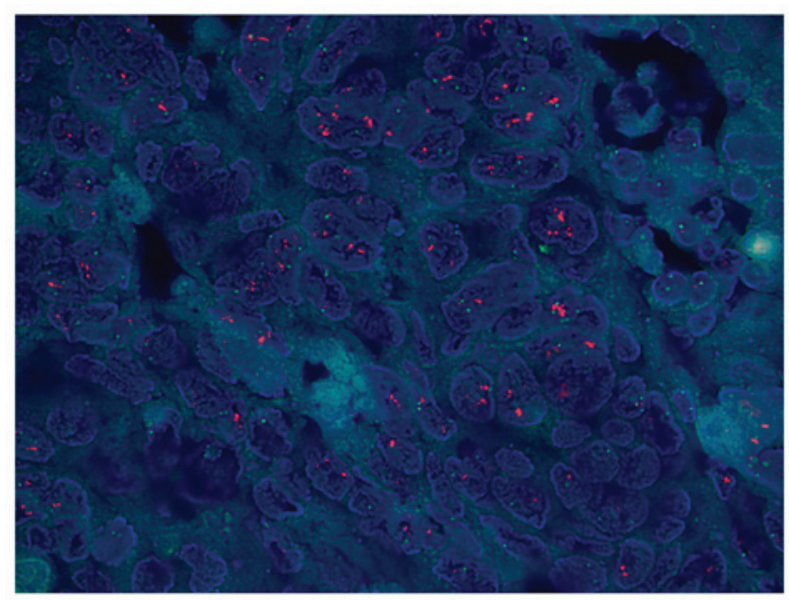

Figure 2. Representative photomicrograph of fluorescent in situ hybridization. The HER2/neu gene was identified in a stomach tumor tissue sample by red fluorescent signals. Chromosome 17 centromere was identified by green fluorescent signals. A total of 94 HER2 signals and 46 CSP17 signals were detected in a total of 30 cells. The HER2:CSP17 ratio was 2.043

occurring simultaneously in different parts of the same patient, or different primary histological malignancies. Diagnosis of multiple primary cancers is still accorded to the international standards developed by Warren and Gates (1) in 1932: i) Each malignant tumor must be histologically confirmed by pathology; ii) each tumor must specifically have their own pathological morphology; iii) tumor metastasis occurring from the different sites or organs of the patient must be excluded. In 1979, Liu et al (3) proposed that primary cancer types may occur in different parts of the body, no correlation exists with each other and each tumor has its unique method of metastasis. The patient in the present study exhibited two independent malignant tumors, each having unique characteristics confirmed by histological and pathological examination, consistent with the diagnosis of primary cancers.

Incidence. The incidence of multiple primary cancers reported in national and international varied. The retrospective survey results of 1,104,269 tumor patients from Demandante et al (2) 


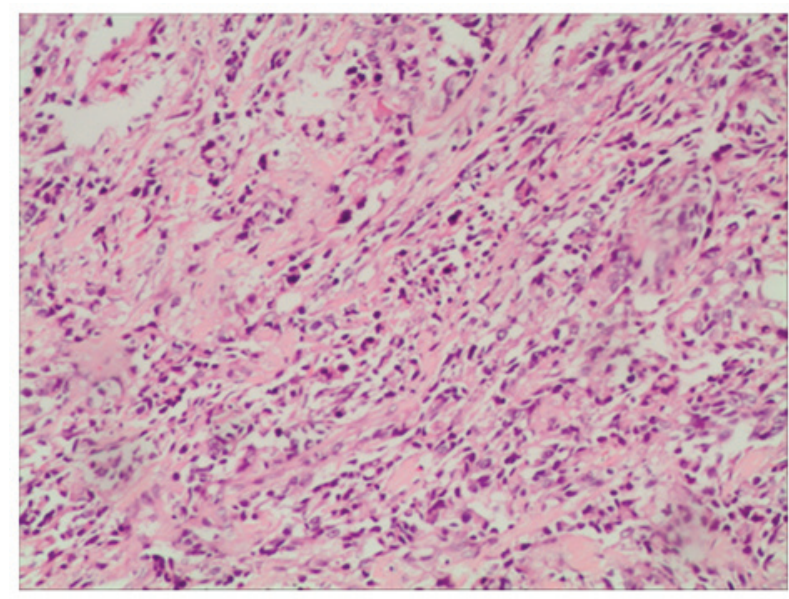

Figure 3. Hematoxylin and eosin staining of right breast tissues. The staining of right breast tissue with hematoxylin and eosin indicated the presence of invasive lobular carcinoma. Small and round cancer cells infiltrated the breast tissue (magnification, $\mathrm{x} 200$ ).

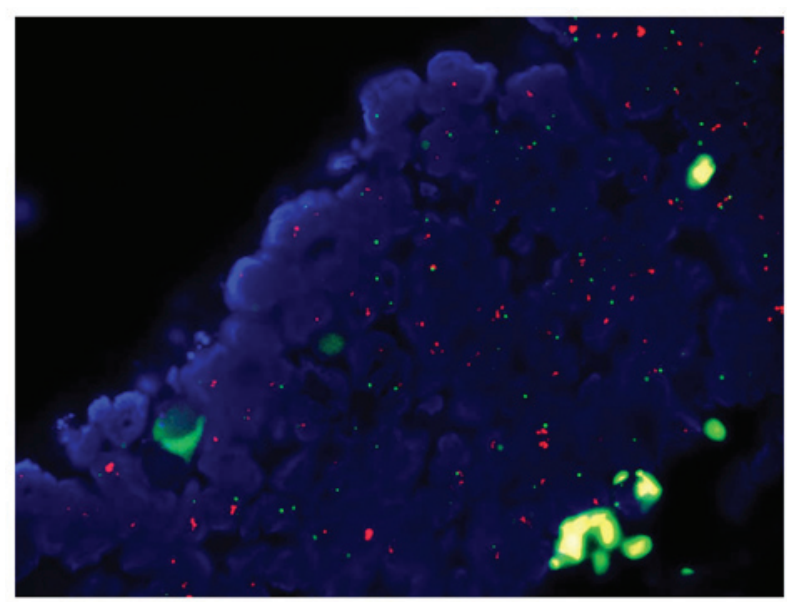

Figure 4. Representative photomicrographs of fluorescent in situ hybridization.. The HER2/neu gene was identified in a breast tumor tissue section by red fluorescent signals. Chromosome 17 centromere was identified by green fluorescent signals. A total of 163 HER2 signals and 72 CSP17 chromosome signals were detected in a total of 30 cells. The HER2:CSP17 ratio was 2.28 .

suggested that the incidence of multiple primary cancers is between 0.73 and $11.7 \%$, with higher prevalence in the elderly. The incidence rate of double primary cancers has increased in recent years, which may be associated with the improvement of the technology used for diagnosis of the primary cancer, the clinical application of anticancer medicines to prolong survival period of patients with the initial primary cancer and increased incidence of second primary cancer. The survey of 63 cases of multiple primary cancers from Irimie et al (4) revealed that women and men occupied 54 and 46\%, respectively, and $>90 \%$ patients were $>40$ years of age. The study also found that multiple primary malignant tumors occur more frequently in the ovary-colon, ovary-breast, breast-breast and other parts. Gastric cancer is one of the most common malignant tumor types, which ranks as the third leading cause of cancer-associated mortality in China, and breast cancer is the first malignant neoplasm of females in China. Among these tumors, HER2-positive gastric cancers range between 6 and 35\% (5), and HER2-positive breast cancers range between 20 and 25\% (6). In the present report, poorly differentiated gastric carcinoma and invasive breast carcinoma were confirmed respectively by histopathological examinations, and each tumor was a primary cancer. While gastric and breast cancer are very common cancer types, which easily metastasize, the incidence rate of gastric-breast primary cancer is extremely low. HER2-positive expression simultaneously in primary breast and primary gastric cancer has not been reported previously in national and international literatures, and to the best of our knowledge, the present study is the first report.

Etiology. Various causes of multiple primary cancers are known and are predominantly associated with predisposition, environmental factors and the host immune factors (7). By contrast, genetic defects are important in causing multiple primary cancers, including Li-Fraumei syndrome, since the abnormality of p53 gene leads to loss-of-function of the tumor suppressor, which eventually leads to the formation of multiple primary cancers in the digestive tract. The same mutation can lead to different tumor types, for example c-RNA virus can cause lymphomas, leukemia and liver cancer, $H$. pylori infections can cause mucus-associated gastric cancer and gastric lymphoma. Thirdly, patients with multiple primary cancers tend to be susceptible and sensitive to carcinogenic factors. If the carcinogenic factors are not ruled out, they may cause cancer in other parts of the body. Other reasons, including the use of chemical agents, endocrine factors and irregular application of chemotherapy may also serve important roles in the development of multiple primary cancers.

Diagnosis and prognosis. A diagnosis of multiple primary cancers may be missed or easily be misdiagnosed (8). Identification of recurrence or metastasis of the first primary cancer from the second primary carcinoma is difficult. It was reported that $50-70 \%$ of the second primary cancer occurs during 3 years after the first primary cancer has been diagnosed. This duration was also the period of the first primary cancer to reoccur and metastasize frequently.

Due to insufficient knowledge or vigilance, clinicians easily misdiagnose the second primary cancer as metastasized or resurrection of the first primary cancer, delaying the treatment of the second primary cancer. Alerting metastasis of the primary cancer and attention to the differentiation between metastatic cancer and the second primary cancer are important strategies for reducing misdiagnosis and missed diagnosis. The effect of treatment for multiple primary cancers is better compared with that of recurrence or metastatic primary cancer, and they are more likely to be cured; therefore physicians must be highly vigilant against the occurrence of multiple primary cancers to improve the effect of the treatment.

HER 2 expression in breast/gastric cancer. In the present case, positive HER2 expression in gastric and breast cancer specimens were detected by FISH. HER2 is an important human epidermal growth factor receptor, and its encoding gene is located at 17q12-21. HER2 protein belongs to a transmembrane protein and its structure is similar to epidermal growth factor receptor, the cytoplasmic domain has tyrosine kinase activity. HER 2 protein is normally inactive and only when binding with specific ligands, the tyrosine kinase is activated, which 
is predominantly caused by receptor dimerization and autophosphorylation of its cytoplasmic domain. HER2 receptor mediates multiple signal transduction pathways including the RAS/RAF/MEK/ERK/RSK and PI3K/PIP2/PIP3/PDK1/AKT pathways. HER2-regulated intracellular signal transduction pathways are involved in the inhibition of apoptosis, the maintenance and promotion of cancer cell growth, tumor angiogenesis, enhanced migration and invasion of tumor cells and destruction of the body's resistance barrier (9). Previous studies have shown that overexpression of HER2 is associated with breast, stomach, ovarian and prostate cancer. Breast cancer patients with overexpression of HER2 have early recurrence, short survival time and poor prognosis (10). HER2 is also as a therapeutic target for breast cancer, and patients with breast cancer patients with positive HER2 expression can clearly benefit from treatment using HER2 monoclonal antibody (11). HER2 is important not only in breast cancer. Its role in the development of gastric cancer is beginning to emerge. A previous study from Oya et al (12) showed that detection of HER2 protein levels can be used as a basis for assessment of prognosis in patients with cancer. According to a previous study on advanced gastric cancers with positive HER2 expression, treatment using the anti-HER2 monoclonal antibody can extend the overall survival of patients to 16 months (13); however, certain studies reported no correlation between HER2 status and prognosis of gastric cancer $(14,15)$. In 2012 , a comprehensive review analyzed the association between the overexpression of HER2 and the prognosis of gastric cancer. Conclusions indicated that HER2 positive expression may be associated with a poor prognosis in patients with gastric cancer (16). Therefore, the expression of HER2 is now considered to be associated with lower survival rate of gastric cancer. HER2 can also be used as a therapeutic target in gastric cancer, currently anti-HER2 therapy is the standard treatment for gastric cancer patients with positive HER2.

In the present case, the patient was initially diagnosed with stomach carcinoma. Following radical surgery, postoperative pathological analysis revealed the full-thickness of the gastric wall and lymph nodes were extensively invaded, and vascular tumor thrombi were observed, implicating poor prognosis. Postoperative chemotherapy was performed, but after half a year, a lump was found in her right breast and a simple lump excision was performed. Postoperative radiotherapy of supraclavicular lymph nodes, chest wall and axillary cavity were executed. For this patient, the expression of HER 2 in primary gastric cancer and primary breast cancer were positive, and diagnosed as simultaneous HER2-positive double primary gastric and breast cancer, which met the categories of the targeted therapy. The patient failed to comply with doctor's prescription and the patient only completed three cycles of Trastuzumab targeted therapy. The patient succumbed to aggressive progression of the disease one year after radical gastrectomy for gastric cancer and half a year after breast lumpectomy.

Although this patient failed to finish target therapy, standardized and regular treatment, including target therapy, must be initiated as early as possible. In clinical practice, for patients with HER2-positive breast cancer, physicians must pay attention to whether lesions exist in the stomach. By contrast, for HER2-positive gastric cancer, breast-associated examinations must be performed in female patients, and if any lesions are found, biopsy and HER2 testing are required in a timely manner to avoid misdiagnosis and the delay of treatment.

\section{Acknowledgements}

The present study was supported by the Science and Technology Program from Changsha City (grant no. 23421).

\section{References}

1. Warren S: Multiple primary malignant tumors. Am J cancer 16: 1358-1414, 1932

2. Demandante CG, Troyer DA and Miles TP: Multiple primary malignant neoplasms: Case report and a comprehensive review of the literature. Am J Clin Oncol 26: 79-83, 2003.

3. Liu F: Double primary carcinomas of the esophagus and stomach: a pathological study of 9 cases. Acta Academiae Medicinae Sinicae 1: 67-70, 1979.

4. Irimie A, Achimas-Cadariu P, Burz C and Puscas E: Multiple primary malignancies-epidemiological analysis at a single tertiary institution. J Gastrointestin Liver Dis 19: 69-73, 2010.

5. Yonemura Y, Ninomiya I, Ohoyama S, Kimura H, Yamaguchi A, Fushida S, Kosaka T, Miwa K, Miyazaki I and Endou Y: Expression of c-erbB-2 oncoprotein in gastric carcinoma. Immunoreactivity for c-erbB-2 protein is an independent indicator of poor short-term prognosis in patients with gastric carcinoma. Cancer 67: 2914-2918, 1991.

6. Engel RH and Kaklamani VG: HER2-positive breast cancer: Current and future treatment strategies. Drugs 67: 1329-1341, 2007.

7. Ray P, Sharifi R, Ortolano V and Guinan P: Involvement of the genitourinary system in multiple primary malignant neoplasms: A review. J Clin Oncol 1: 574-581, 1983.

8. Babacan NA, Aksoy S, Cetin B, Ozdemir NY, Benekli M, Uyeturk U, Ali Kaplan M, Kos T, Karaca H, Oksuzoglu B, et al: Multiple primary malignant neoplasms: Multi-center results from Turkey. J BUON 17: 770-775, 2012.

9. Tai W, Mahato R and Cheng K: The role of HER2 in cancer therapy and targeted drug delivery. J Control Release 146: 264-275, 2010.

10. Pils D, Pinter A, Rerbenwein J, Alfanz A, Horak P, Schmid BC, Hefler L, Horvat R, Reinthaller A, Zeillinger R and Krainer M: In ovarian cancer the prognostic influence of HER2/neu is not dependent on the CXCR4/SDF-1 signalling pathway. Br J Cancer 96: 485-491, 2007.

11. Scherbakov AM, Krasil'nikov MA and Kushlinskii NE: Molecular mechanisms of hormone resistance of breast cancer. Bull Exp Biol Med 155: 384-395, 2013.

12. Oya M, Yao T and Tsuneyoshi M: Expressions of cell-cycle regulatory gene products in conventional gastric adenomas: Possible immunohistochemical markers of malignant transformation. Hum Pathol 31: 279-287, 2000.

13. Van Cutsem E, Kang Y, Chung H, Shen L, Sawaki A, Lordick F, et al: Efficacy results from the ToGA trial: A phase III study of trastuzumab added to standard chemotherapy in first-line HER2-positive advanced gastric cancer. J Clin Oncol 27: 15, 2009.

14. Kunz PL, Mojtahed A, Fisher GA, Ford JM, Chang DT, Balise RR, Bangs CD, Cherry AM and Pai RK: HER 2 expression in gastric and gastroesophageal junction adenocarcinoma in a US population: Clinicopathologic analysis with proposed approach to HER2 assessment. Appl Immunohistochem Mol Morphol 20: 13-24, 2012.

15. Moelans CB, Milne AN, Morsink FH, Offerhaus GJ and Van Diest PJ: Low frequency of HER2 amplification and overexpression in early onset gastric cancer. Cell Oncol (Dordr) 34: 89-95, 2011

16. Jørgensen JT and Hersom M: HER2 as a prognostic marker in gastric cancer-a systematic analysis of data from the literature. J Cancer 3: 137-144, 2012. 